\title{
Entanglement Spheres and a UV-IR Connection in Effective Field Theories
}

\author{
Natalie Klco $\circledast^{1, *}$ and Martin J. Savage $\circledast^{2, \dagger}$ \\ ${ }^{1}$ Institute for Quantum Information and Matter (IQIM) and Walter Burke Institute for Theoretical Physics, \\ California Institute of Technology, Pasadena, California 91125, USA \\ ${ }^{2}$ InQubator for Quantum Simulation (IQuS), Department of Physics, University of Washington, \\ Seattle, Washington 98195, USA
}

(Received 27 April 2021; accepted 27 September 2021; published 16 November 2021)

\begin{abstract}
We show that long-distance quantum correlations probe short-distance physics. Two disjoint regions of the latticized, massless scalar field vacuum are numerically demonstrated to become separable at distances beyond the negativity sphere, which extends to infinity in the continuum limit. The size of this quantum coherent volume is determined by the highest momentum mode supported in the identical regions, each of diameter $d$. More generally, effective field theories (EFTs), describing a system up to a given momentum scale $\Lambda$, are expected to share this feature-entanglement between regions of the vacuum depends upon the UV completion beyond a separation proportional to $\Lambda$. Through calculations extended to three dimensions, the magnitude of the negativity at which entanglement becomes sensitive to UV physics in an EFT (lattice or otherwise) is conjectured to scale as $\sim e^{-\Lambda d}$, independent of the number of spatial dimensions. It is concluded that two-region vacuum entanglement at increasing separations depends upon the structure of the theory at increasing momentum scales. This phenomenon may be manifest in perturbative QCD processes.
\end{abstract}

DOI: 10.1103/PhysRevLett.127.211602

Introduction.-Fundamental principles of effective field theories (EFTs) leverage clear separations of energy scales to identify relevant degrees of freedom and to build a systematically improvable hierarchy of local operators. By incorporating all relevant interactions consistent with the symmetries of the theory, this hierarchy accurately captures physics in regimes where scale ratios are small (see, for example, Ref. [1]). While short-distance properties require high-energy probes (e.g., the exploration of hadronic structure through deep inelastic scattering) or quantum fluctuations (e.g., flavor-changing neutral currents), longdistance properties may be informed by infrared (IR) observables at low energies. Thus, long-distance physics tends to be insensitive to ultraviolet (UV) modifications incorporated in an EFT through momentum truncations or the "integrating out" of high-energy degrees of freedom. In this Letter, it is shown that the distillable entanglement between two disjoint regions of a massless scalar field is a long-distance observable sensitive to the treatment of the UV degrees of freedom. In particular, a finite momentum truncation, limiting the effective information resolution of the field, will cause distantly separated spatial regions of

Published by the American Physical Society under the terms of the Creative Commons Attribution 4.0 International license. Further distribution of this work must maintain attribution to the author(s) and the published article's title, journal citation, and DOI. Funded by SCOAP . the field to not only exhibit vanishing distillable entanglement, but to become separable. As such, momentum-space regularizations in the UV necessarily introduce an IR truncation of the vacuum quantum correlations [2-9] at large spatial distances, limiting the IR regime of EFT validity from the perspective of quantum mechanical inseparability.

Quantum field theory (QFT) has provided a natural unifying perspective of particles as excitations or localized packets of energy embedded in fundamental fields. The many successes calculating entanglement in QFT benefit from an assortment of powerful strategies through lattices, replica tricks, holography, and the AdS/CFT correspondence, e.g., [10-29]. Despite these heroic developments, particular parameter regimes, e.g., the distillable entanglement between regions distantly separated compared to their size, have evaded analytic control, retaining the importance of numerical explorations.

While the QFT description has been remarkably successful experimentally, conclusions about the underlying structure of nature are limited by the possibility that this success may result from the fact that any relativistic quantum system with Lorentz symmetry and cluster decomposition at long distances will behave as a quantum field at low energies $[1,30]$. In fact, considerations in large volumes, inspired by entropy being nonextensive in black hole thermodynamics [31-35], has led to a conjecture that the QFT description egregiously overcounts degrees of freedom [36,37]. This perspective has inspired the proposal 
of a relationship between UV and IR truncations in the valid regime of an EFT-limiting the volume to avoid the extensively scaling EFT entropy from exceeding that of a potential black hole [38,39]. Furthermore, mixing between UV and IR physics has been connected to properties of nonlocality and noncommutative or gravitational generalizations of quantum fields, e.g., [40-46].

This Letter demonstrates a connection between UV and IR physics with an observation that the entanglement in the vacuum of a simple quantum field, the massless noninteracting scalar field, is sensitive to high-momentum modes at large spatial separations. This extends to mixed states the speculation of Ref. [13], informed by the modewise spatial entanglement structure of harmonic chain bipartite pure states, on the role of short-wavelength modes in the persistence of vacuum entanglement. Explicitly, we have extended numerical lattice computations of the logarithmic negativity between pixelated spherical regions to three dimensions, allowing identification of a dimensionindependent scaling of the smallest supported negativity. Such extensions to higher dimension are nontrivial due to cancellations between polynomial- and logarithmic-scaling lattice correlation functions generating an exponentially small entanglement, manifesting as a sign problem in the lattice basis. In the process of exploring the entanglement structure of these systems, we have provided numerical evidence, through a separability flow [47], that regions separated beyond the negativity sphere are also separable, precluding the presence of entanglement with positive partial transpose (PPT) in the massless lattice scalar field. The array of calculations presented in this Letter serve as an explicit example of a more general relationshiplong-distance quantum correlations probe short-distance physics - broadly affecting the regime of validity for quantum observables in EFTs.

Vacuum entanglement sphere.-As a necessary condition for separability, the negativity [48-51] quantifies the violation of partial transposition-locally negating the momentum in one region for continuous variables [50] - from producing a physical density matrix (non-negative eigenvalues). If a quantum state is separable across a bipartition, partial transposition is a map that preserves the positivity of the density matrix (PPT). Violations to this positivity herald inseparability and thus the presence of entanglement for both mixed and pure quantum states.

Consider the matrix of two-point correlation functions,

$$
G_{i, j}=\left\langle\phi_{i} \phi_{j}\right\rangle, \quad H_{i, j}=\left\langle\pi_{i} \pi_{j}\right\rangle,
$$

where $\{i, j\} \in A \cup B$ for two local field regions $A$ and $B$. For $n_{s}$ sites in each region, $G$ and $H$ are $\left(2 n_{s} \times 2 n_{s}\right)$ dimensional matrices with matrix elements controlled by $\mathbf{n}$, the vector separating sites $\{i, j\}$. In the thermodynamic limit, the integral representation of the modified Bessel function of the first kind $I_{\nu}(z)$ allows a succinct calculation of the necessary correlators as

$$
G(\mathbf{n})=\frac{1}{\sqrt{\pi}} \int_{0}^{\infty} d x e^{-\left(m^{2}+2 \mathrm{D}\right) x^{2}} \prod_{i} I_{n_{i}}\left(2 x^{2}\right)
$$

and

$$
\begin{aligned}
H(\mathbf{n})= & \left(m^{2}+2 \mathrm{D}\right) G(\mathbf{n})-\sum_{\left\{\mathbf{n}^{\prime}\right\}} G\left(\mathbf{n}^{\prime}\right) \\
& \rightarrow m^{2} G(\mathbf{n})-\nabla^{2} G(\mathbf{n}),
\end{aligned}
$$

where $\left\{\mathbf{n}^{\prime}\right\}$ is the set of $2 D$ integer vectors shifted by \pm 1 in each direction of the $D$-dimensional lattice (see Sec. S1 of the Supplemental Material [52] for further details). The logarithmic negativity is additive

$$
\mathcal{N}=-\sum_{i=1}^{2 n_{s}} \log _{2} \min \left(\nu_{i}^{\Gamma}, 1\right)
$$

where $\nu_{i}^{\Gamma}$ are the symplectic eigenvalues of the partially transposed covariance matrix, which may be calculated as the eigenvalues of $2 \sqrt{G H^{\Gamma}}[13,17,55]$. The superscript $\Gamma$ indicates partial transposition of the conjugate momentum two-point functions and may be implemented, in practice, by negating the matrix element of $H_{i, j}$ if the sites at positions $\{i, j\}$ are in different regions $\{A, B\}$ of the field [50].

Numerical evaluations of the negativity between disjoint regions of the massless scalar field have shown that the negativity in the continuum limit with $\tilde{r} \gg d$ decays exponentially as $\mathcal{N} \sim e^{-\beta(\tilde{r} / d)}$, with $\tilde{r}$ the separation between the field regions and $d$ the diameter of each region. Extractions of the negativity decay constant yield $\beta_{1 \mathrm{D}}=2.82(3) \sim 2 \sqrt{2}$ [17], $\beta_{2 \mathrm{D}}=5.29(4)$ [55], and we currently estimate $\beta_{3 \mathrm{D}}=7.6(1)$. Section $\mathrm{S} 3$ of the Supplemental Material [52] provides further discussion of this three-dimensional calculation. As proposed in Ref. [55], this progression with dimensionality is consistent with $\beta_{D} \sim D$, the negativity becoming increasingly localized in higher dimensions. At a finite lattice spacing, where regions experience finite pixelation, there exists a nonperturbative death of negativity at large separation, $\tilde{r}_{\not / x}$ $[12,13,16,17,20,23-26,28,29]$. The scaling of this negativity sphere with the region pixelation was previously found to be $\tilde{r}_{\not \gamma / d} / d \sim(\gamma / a) d$, with $\gamma_{1 \mathrm{D}}=1.114(2), \gamma_{2 \mathrm{D}}=0.60(2)$ [55], and we currently estimate $\gamma_{3 \mathrm{D}}=0.43(2)$. With a dependence of $\gamma_{D} \sim D^{-1}$, a more stringent negativity sphere is observed with increasing spatial dimension.

The negativity is not generally a necessary and sufficient condition for determining the separability of Gaussian states. In particular, there is a form of nondistillable entanglement, bound entanglement [56], that may forbid 


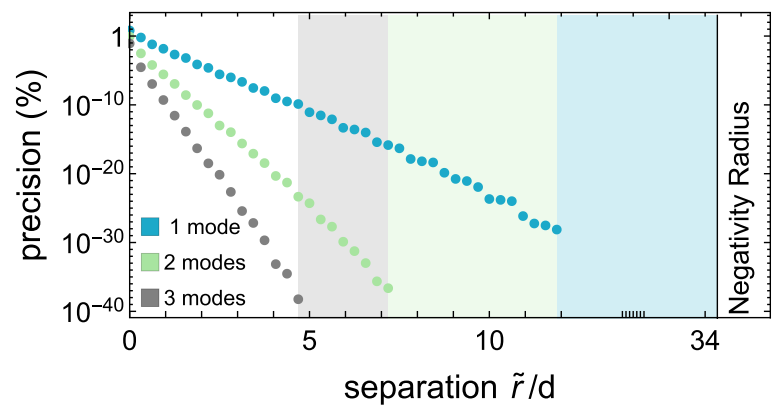

FIG. 1. Precision of the mode-restricted logarithmic negativity as a function of spatial separation in the one-dimensional massless scalar field with region diameter $d=32$. With increasing separation, the discrepancy vanishes and the entirety of the negativity is captured by the lowest few eigenmodes of $G H^{\Gamma}$, indicated by the shaded background.

separability while avoiding detection by the negativity criterion [57-62]. By employing the necessary and sufficient Gaussian state separability criterion of Ref. [47], which acts as a flow maintaining the separability (or nonseparability) of covariance matrices, while systematically simplifying the entanglement structure, it is found that regions of the scalar field outside the negativity sphere are separable. The negativity sphere is thus promoted to an entanglement sphere and describes a finite-sized quantum mechanically coherent volume between regions of the field (see Sec. S2 of the Supplemental Material [52] for further discussion). As such, any observable calculated outside the entanglement sphere will be characterized by factorizable classical probability distributions. For example, the mutual information, which persists outside this entanglement sphere, is there quantifying correlations that are entirely classical.

To the extent that spin models generically are known to exhibit vanishing two-site entanglement beyond a finite site separation [63], the presence of the entanglement sphere is not without precedence. One intriguing implication, however, is that the long-distance entanglement known to be present in the continuum quantum field is necessarily captured through the presence of genuine high-body entanglement and a Borromean structure on the latticeregions entangled at long distances may contain vanishing negativity for all smaller subsets of sites spanning the two regions. This reliance on genuine high-body entanglement at large distances is intuitively consistent with the complexity of low-pixelation regions being insufficient to support long-distance entanglement.

While not exact and currently limited in numerical precision due to the presence of a sign problem exacerbated in higher dimensions, the calculated dependence of the negativity decay and size of the entanglement sphere indicate that the emergence of separability at large distances is set by the UV truncation of the theory. In particular, at the surface of the entanglement sphere, the minimum value of the negativity supported by the field before separability occurs is $\mathcal{N}_{\not j} \sim e^{-\beta_{D}\left(\tilde{r}_{j} / d\right)} \sim e^{-\beta_{D} \gamma_{D} d / a}$. Combining the calculations above to inform the product provides $\quad \beta_{1 \mathrm{D}} \gamma_{1 \mathrm{D}}=3.14(3), \quad \beta_{2 \mathrm{D}} \gamma_{2 \mathrm{D}}=3.2(1), \quad$ and $\beta_{3 \mathrm{D}} \gamma_{3 \mathrm{D}}=3.2(1)$. The stability of this product with spatial dimension leads us to conjecture that

$$
\mathcal{N}_{\not \supset \mid} \sim e^{-\beta_{D} \gamma_{D} d / a} \sim e^{-\frac{\pi d}{a}} \sim e^{-\Lambda d},
$$

where $\Lambda$ is the scale of the UV truncation, independent of the number of spatial dimensions. For disjoint regions of the vacuum, the threshold negativity below which the field becomes separable is determined by the diameter of each region and the highest allowed momentum mode. In two dimensions, where $\pi d$ acts as the circumference of the circular field regions, the scaling of the negativity at the entanglement sphere is coincidentally consistent with an area dependence. Though the negativity radius has been conventionally interpreted as a lattice artifact of no consequence to continuum physics, this conjecture indicates that a truncation in the distillable entanglement will be present at long distances in continuum theories with finite UV truncations.

Entanglement sphere and region momentum.-A tangible understanding of the UV-IR connection found in the distillable entanglement at large distances can be elucidated

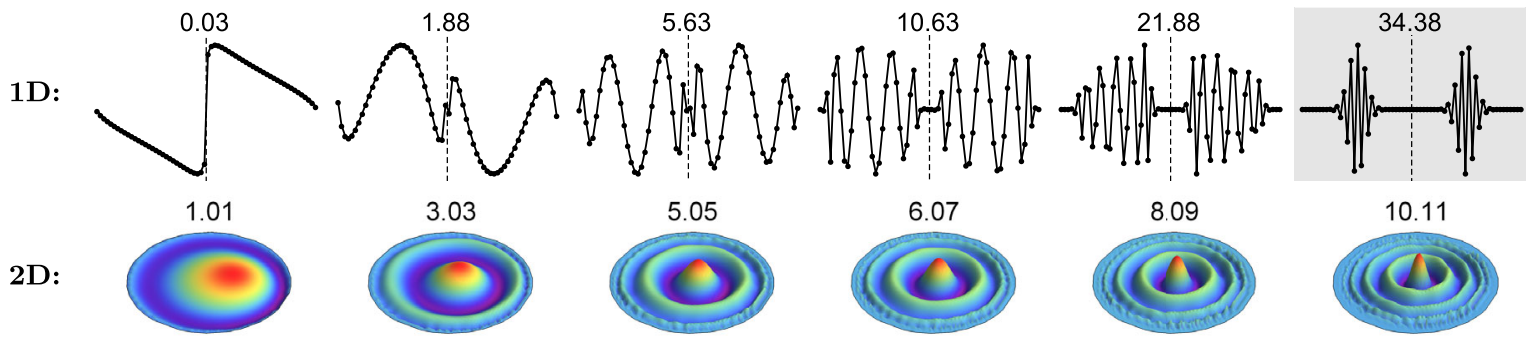

FIG. 2. Negativity ground-state wave functions of $G H^{\Gamma}$ in the massless scalar field in one (top) and two (bottom) spatial dimensions. The 1D wave functions are provided across the two regions separated at indicated distances $\tilde{r} / d$ with $d=n_{s}=32\left(\tilde{r}_{\not j /} / d=34.34\right)$. The 2D wave functions are shown for one region (negative spatial parity as in 1D) with $d=64$ for a variety of $\tilde{r} / d$ separations within the entanglement sphere, $\tilde{r}_{\not \supset d} / d \sim 18$. 


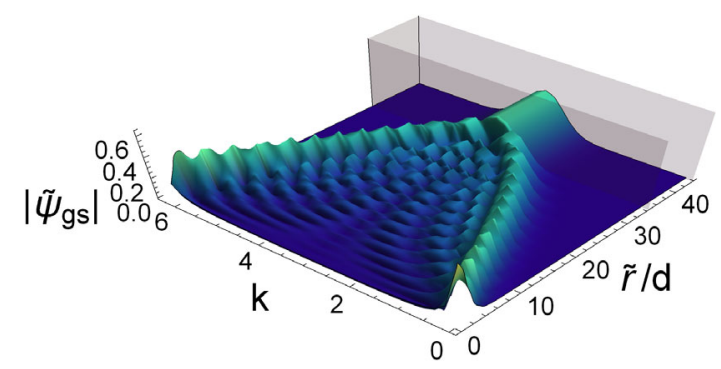

FIG. 3. Momentum-space negativity ground-state wave functions of $G H^{\Gamma}$ for the 1D massless scalar field isolated to a single region of $n_{s}=32$ as a function of $\tilde{r} / d$ region separation. The gray area at large separation lies outside the negativity radius, $\tilde{r}_{\not x} / d=34.34$.

through examination of the dominant $G H^{\Gamma}$ right eigenvector contributing to the logarithmic negativity of Eq. (4). While many symplectic eigenvalues contribute to the negativity at small separations, this number diminishes as the separation increases, as shown in Fig. 1. As the separation approaches the entanglement sphere, the negativity is characterized by the single ground state of $G H^{\Gamma}$. In Ref. [55], we examined the negativity ground-state wave function and found it to evolve to high-momentum components with increasing separation. The structure of the $G H^{\Gamma}$ ground state within the field regions at increasing separation is shown in Fig. 2 for one and two spatial dimensions. In 1D, the ground state is demonstrated to have negative parity between the two regions, allowing the depiction of a single region in $2 \mathrm{D}$ for visual clarity. At small separations, the negativity ground state is composed of low-frequency contributions. As the separation grows to multiple region diameters, fluctuations sequentially emerge from the boundary and propagate toward the central peak. These fluctuations comprise high-frequency contributions and stress the pixelation of the region. In this long-distance regime, the region negativity ground-state wave function tends to become rotationally symmetric with a boundary condition vanishing at the edges, allowing the characterization of region entanglement to become effectively onedimensional.

The frequency space representations of the $1 \mathrm{D}$ region wave functions, $\tilde{\psi}(k)=\left(1 / \sqrt{n_{s}}\right) \sum_{x} \psi(x) e^{i k x}$, are shown in Fig. 3 for a Brillouin zone $k \in\left(2 \pi / n_{s}\right) \mathbb{Z}_{n_{s}}$. At small separations, support in momentum space is localized to the regimes of small region momentum at the 0 and $2 \pi$ boundaries of the Brillouin zone. As the separation between regions increases, the momentum content within the region is driven to the high-momentum boundary of $k=\pi$. Outside the entanglement sphere (gray region), the momentum-space wave function, now separable, locally saturates the UV truncation and ceases to evolve.

Implications for effective field theories.-Effective field theories provide a powerful technique for computing lowenergy processes in systems with hierarchies in their energy spectrum. The lore is that the predictions of an EFT (valid below some momentum scale $\Lambda$ ) can systematically reproduce those of the full theory (UV complete) when the IR degrees of freedom and symmetries match. When the full theory is known, local counterterms in the EFT are determined by matching matrix elements computed in both theories. The results of lattice calculations of the negativity discussed in the previous sections indicate that the behaviors of distillable entanglement and separability at long distances of a massless noninteracting scalar field are determined by high-momentum modes of the field, with the radius of the entanglement sphere determined by the UV cutoff. In particular, the exponentially small negativity between regions of vacuum is lost beyond some dimensionless separation determined by the maximum momentum mode allowed by the lattice spacing $\pi / a$. However, this feature is generic for any low-energy EFT valid up to some momentum scale $\Lambda$. The higher the EFT cutoff, the larger the separation between regions of the EFT vacuum that remain entangled.

The lattice and other abrupt cutoffs in momentum space lead to a well-defined distance, beyond which regions of the vacuum state are separable. Other regulators can be used to render EFT computations finite by implementing a form factor in momentum or position space, e.g., PauliVillars (PV) or dimensional regularization (DR). These provide smooth modifications of perturbative Feynman diagrams, which furnish finite loop diagrams, along with associated counterterms that depend upon the PV mass or the DR scale $\mu$. As calculations that can be compared with experiment are independent of the choice of regulator and renormalization scheme, it is the UV completion of the theory that will determine the behavior (vanishing or otherwise) of negativity at long distances (see Sec. S4 of the Supplemental Material [52]). In this way, precision studies of the large-scale entanglement in the quantum vacuum can probe short-distance physics. However, the precise translation of such entanglement studies into constraints on, for instance, beyond the standard model physics, remains to be explored.

Rather than an IR constraint on the spatial volume scaling with the inverse UV truncation $L \sim \Lambda^{-3}$ as associated with fundamental bounds on black hole entropy [38,39], this Letter identifies a linearly scaling IR truncation, $L \sim \Lambda$, necessary to accurately capture the inseparability of the field ground state. While the core principles of these cutoffs lie in the saturation of spatially localized information, their distinct scaling suggests a stringency crossover - the formation of black holes being the relevant constraint for UV truncations above the crossover and the separability criterion being the relevant constraint for UV truncations below. To gain some insight into the potential impacts, we provide mass and length scales for two different scenarios. Using, in three dimensions, $\tilde{r}_{\not, j} \sim d^{2} /(3 a) \rightarrow \Lambda d^{2} /(3 \pi)$, and setting $\Lambda=M_{\mathrm{Pl}}$ to be 
the Planck mass, we find that regions of vacuum of a massless noninteracting scalar field approximately the size of the proton are separable at distances beyond $\sim 6 \mathrm{~km}$, and that regions approximately the size of an atom are separable beyond $\sim 10^{11} \mathrm{~km}$. If the cutoff of the EFT is instead $\Lambda \sim 1 \mathrm{TeV}$, then proton-sized regions are separable beyond $\sim 500 \mathrm{fm}$ and atomic-sized regions beyond $\sim 5 \mathrm{~mm}$. Evaluating the potential for possible signatures from these distance scales in experiment, one takes pause from the values of negativity at the point of separability, which are $\sim 10^{-1800}$ and $\sim 10^{-2 \times 10^{8}}$, respectively, for a $\mathrm{TeV}$ scale cutoff. The magnitude of this effect falls exponentially with separation, imposing what are likely to be severe limits on the constraints that can be determined by experiment.

Discussion.-By considering massless, noninteracting scalar field theory, the onset of separability between regions of a quantum vacuum in discretized systems has been shown to result from a connection between long- and shortdistance physics. In particular, the maximum distance between regions of the vacuum that are entangled is directly related to the high-momentum modes of the field. For a lattice field theory, this distance is set by the inverse lattice spacing. While analytic results and a deep understanding of the underlying mechanism(s) remain to be uncovered, numerical explorations indicate that the onset of separability is connected to a saturation of information contained in regions of the pixelated vacuum. This naturally suggests that such effects are present in the vacuum of any quantum field theory that has a UV cutoff, as in the case of any EFT. This violates EFT lore, which assumes that IR physics in a complete theory can be systematically recovered in a lowenergy EFT. The present results show, however, that a UV cutoff in an EFT leads to separable regions of the vacuum beyond an entanglement sphere. Further lattice calculations are required to reduce uncertainties associated with the negativity parameters and to pursue calculations in higher spatial dimensions to better define their dimensional scaling.

For most systems, the discrepancies in measures of entanglement are very small, with estimates suggesting they will be challenging to explore experimentally. However, recent advances in the control of quantum systems capable of representing bosonic fields, e.g., [6467] and the potential to leverage quantum computing technologies as quantum detectors of background field entanglement properties yields numerous directions for potential experimental connection. Beyond the vacuum, experimental signatures of this UV-IR connection, and its implications for low-energy EFTs, may be more profitably sought in excited states of the field theory, in particular, in systems of two or more wave packets, themselves amenable to detection and measurement. For example, in the EFT description of $S$-channel scattering of nuclei, entanglement power has been proposed to impact the relevant hierarchy of local operators $[46,68]$.

While fundamental massless scalar fields do not exist in nature, fields with massless or light excitations and modest or weak interaction strengths, e.g., electromagnetism, perturbative QCD, nuclear EFTs around the chiral limit $[69,70]$, gravity, axions, Bose-Einstein condensates (e.g., Refs. [71,72]), and neutrinos, may exhibit a modified vacuum entanglement structure at long distances due to UV physics. High-energy processes in the nucleon or nucleus, probing distances below the confinement scale with a spatial momentum transfer $\mathbf{Q}$ and UV cutoff $\Lambda$, may be sensitive [73-77] to such modifications for $\tilde{r}_{\not>\lambda} \gtrsim \Lambda /\left(3 \pi|\mathbf{Q}|^{2}\right)$, resulting in a negativity deviation $\sim \exp (-\Lambda /|\mathbf{Q}|)$. Similar signatures are also expected in massive fields, where the long-distance negativity decay becomes Gaussian [17]. It would appear that the phenomenology of entanglement in the quantum vacuum at long distances may depend on what lies beyond the standard model describing electroweak and strong interactions.

We would like to thank Silas Beane, Douglas Beck, Roland Farrell, David Kaplan, Aidan Murran, John Preskill, and Alessandro Roggero for valuable discussions. We have made extensive use of Wolfram Mathematica [78] and the ADVANPIX multiprecision computing toolbox [79] for MATLAB [80]. Numerical results are available upon request. N. K. is supported in part by the Walter Burke Institute for Theoretical Physics, and by the U.S. Department of Energy Office of Science, Office of Advanced Scientific Computing Research (Award No. DE-SC0020290) and Office of High Energy Physics DE-ACO2-07CH11359. M. J. S. was supported in part by the U.S. Department of Energy, Office of Science, Office of Nuclear Physics, InQubator for Quantum Simulation (IQuS) under Award No. DOE (NP) Award No. DESC0020970.

natklco@caltech.edu

mjs5@uw.edu

On leave from the Institute for Nuclear Theory.

[1] S. Weinberg, Phenomenological Lagrangians, Physica (Amsterdam) 96A, 327 (1979).

[2] H. Reeh and S. Schlieder, Bemerkungen zur unitäräquivalenz von lorentzinvarianten feldern, Nuovo Cimento 22, 1051 (1961).

[3] S. J. Summers and R. Werner, The vacuum violates Bell's inequalities, Phys. Lett. 110A, 257 (1985).

[4] S. J. Summers and R. Werner, Bell's inequalities and quantum field theory. I. General setting, J. Math. Phys. (N.Y.) 28, 2440 (1987).

[5] S. J. Summers and R. Werner, Bell's inequalities and quantum field theory. II. Bell's inequalities are maximally violated in the vacuum, J. Math. Phys. (N.Y.) 28, 2448 (1987). 
[6] A. Valentini, Non-local correlations in quantum electrodynamics, Phys. Lett. A 153, 321 (1991).

[7] B. Reznik, Entanglement from the vacuum, Found. Phys. 33, 167 (2003).

[8] B. Reznik, A. Retzker, and J. Silman, Violating Bell's inequalities in the vacuum, Phys. Rev. A 71, 042104 (2005).

[9] E. Witten, APS medal for exceptional achievement in research: Invited article on entanglement properties of quantum field theory, Rev. Mod. Phys. 90, 045003 (2018).

[10] C. Holzhey, F. Larsen, and F. Wilczek, Geometric and renormalized entropy in conformal field theory, Nucl. Phys. B424, 443 (1994).

[11] C. G. Callan, Jr. and F. Wilczek, On geometric entropy, Phys. Lett. B 333, 55 (1994).

[12] K. Audenaert, J. Eisert, M. B. Plenio, and R. F. Werner, Entanglement properties of the harmonic chain, Phys. Rev. A 66, 042327 (2002).

[13] A. Botero and B. Reznik, Spatial structures and localization of vacuum entanglement in the linear harmonic chain, Phys. Rev. A 70, 052329 (2004).

[14] P. Calabrese and J. L. Cardy, Entanglement entropy and quantum field theory, J. Stat. Mech. (2004) P06002.

[15] S. Ryu and T. Takayanagi, Holographic Derivation of Entanglement Entropy from AdS/CFT, Phys. Rev. Lett. 96, 181602 (2006).

[16] J. Kofler, V. Vedral, M. S. Kim, and Ć. Brukner, Entanglement between collective operators in a linear harmonic chain, Phys. Rev. A 73, 052107 (2006).

[17] S. Marcovitch, A. Retzker, M. B. Plenio, and B. Reznik, Critical and noncritical long-range entanglement in KleinGordon fields, Phys. Rev. A 80, 012325 (2009).

[18] H. Casini and M. Huerta, Entanglement entropy in free quantum field theory, J. Phys. A 42, 504007 (2009).

[19] T. Nishioka, S. Ryu, and T. Takayanagi, Holographic entanglement entropy: An overview, J. Phys. A 42, 504008 (2009).

[20] P. Calabrese, J. Cardy, and E. Tonni, Entanglement entropy of two disjoint intervals in conformal field theory, J. Stat. Mech. (2009) P11001.

[21] P. Calabrese and J. Cardy, Entanglement entropy and conformal field theory, J. Phys. A 42, 504005 (2009).

[22] H. Casini, M. Huerta, and R. C. Myers, Towards a derivation of holographic entanglement entropy, J. High Energy Phys. 05 (2011) 036.

[23] P. Calabrese, J. Cardy, and E. Tonni, Entanglement Negativity in Quantum Field Theory, Phys. Rev. Lett. 109, 130502 (2012).

[24] P. Calabrese, J. Cardy, and E. Tonni, Entanglement negativity in extended systems: A field theoretical approach, J. Stat. Mech. (2013) P02008.

[25] M. Reza Mohammadi Mozaffar and A. Mollabashi, Entanglement in Lifshitz-type quantum field theories, J. High Energy Phys. 07 (2017) 120.

[26] A. Coser, C. De Nobili, and E. Tonni, A contour for the entanglement entropies in harmonic lattices, J. Phys. A 50, 314001 (2017).

[27] P. Ruggiero, E. Tonni, and P. Calabrese, Entanglement entropy of two disjoint intervals and the recursion formula for conformal blocks, J. Stat. Mech. (2018) 113101.
[28] N. Klco and M. J. Savage, Systematically localizable operators for quantum simulations of quantum field theories, Phys. Rev. A 102, 012619 (2020).

[29] G. Di Giulio and E. Tonni, On entanglement Hamiltonians of an interval in massless harmonic chains, J. Stat. Mech. (2020) 033102.

[30] S. Weinberg, What is quantum field theory, and what did we think it is? in Proceedings of the Conference on Historical Examination and Philosophical Reflections on the Foundations of Quantum Field Theory, Boston University, 1996 (1996), pp. 241-251 [arXiv:hep-th/9702027].

[31] J. D. Bekenstein, Black holes and entropy, Phys. Rev. D 7, 2333 (1973).

[32] J. D. Bekenstein, Generalized second law of thermodynamics in black hole physics, Phys. Rev. D 9, 3292 (1974).

[33] S. W. Hawking, Black holes and thermodynamics, Phys. Rev. D 13, 191 (1976).

[34] J. D. Bekenstein, A universal upper bound on the entropy to energy ratio for bounded systems, Phys. Rev. D 23, 287 (1981).

[35] J. D. Bekenstein, Entropy bounds and black hole remnants, Phys. Rev. D 49, 1912 (1994).

[36] G. 't Hooft, Dimensional reduction in quantum gravity, Conf. Proc. C 930308, 284 (1993), https://arxiv.org/abs/grqc/9310026.

[37] L. Susskind, The world as a hologram, J. Math. Phys. (N.Y.) 36, 6377 (1995).

[38] A. G. Cohen, D. B. Kaplan, and A. E. Nelson, Effective Field Theory, Black Holes, and the Cosmological Constant, Phys. Rev. Lett. 82, 4971 (1999).

[39] A. G. Cohen and D. B. Kaplan, Gravitational contributions to the electron $g$-factor, arXiv:2103.04509.

[40] S. Minwalla, M. Van Raamsdonk, and N. Seiberg, Noncommutative perturbative dynamics, J. High Energy Phys. 02 (2000) 020.

[41] A. Matusis, L. Susskind, and N. Toumbas, The IR/UV connection in the noncommutative gauge theories, J. High Energy Phys. 12 (2000) 002.

[42] G. Minton and V. Sahakian, A new mechanism for nonlocality from string theory: UV-IR quantum entanglement and its imprints on the CMB, Phys. Rev. D 77, 026008 (2008).

[43] R. Horvat, A. Ilakovac, J. Trampetic, and J. You, On UV/IR mixing in noncommutative gauge field theories, J. High Energy Phys. 12 (2011) 081.

[44] J. L. Karczmarek and C. Rabideau, Holographic entanglement entropy in nonlocal theories, J. High Energy Phys. 10 (2013) 078.

[45] D. Lust and E. Palti, Scalar fields, hierarchical UV/IR mixing and the weak gravity conjecture, J. High Energy Phys. 02 (2018) 040.

[46] S. R. Beane and R. C. Farrell, Geometry and entanglement in the scattering matrix, Ann. Phys. (Amsterdam) 433, 168581 (2021).

[47] G. Giedke, B. Kraus, M. Lewenstein, and J. I. Cirac, Entanglement Criteria for All Bipartite Gaussian States, Phys. Rev. Lett. 87, 167904 (2001).

[48] M. Horodecki, P. Horodecki, and R. Horodecki, On the necessary and sufficient conditions for separability of mixed quantum states, Phys. Lett. A 223, 1 (1996). 
[49] G. Vidal and R. F. Werner, Computable measure of entanglement, Phys. Rev. A 65, 032314 (2002).

[50] R. Simon, Peres-Horodecki Separability Criterion for Continuous Variable Systems, Phys. Rev. Lett. 84, 2726 (2000).

[51] M. B. Plenio, Logarithmic Negativity: A Full Entanglement Monotone That is Not Convex, Phys. Rev. Lett. 95, 090503 (2005).

[52] See Supplemental Material at http://link.aps.org/ supplemental/10.1103/PhysRevLett.127.211602 for entanglement spheres and a UV-IR connection in effective field theories, which includes Refs. [53,54].

[53] G. Giedke, L.-M. Duan, J. Ignacio Cirac, and P. Zoller, Distillability criterion for all bipartite Gaussian states, Quantum Inf. Comput. 1, 79 (2001).

[54] DLMF, NIST Digital Library of Mathematical Functions, edited by F. W. J. Olver, A. B. Olde Daalhuis, D. W. Lozier, B. I. Schneider, R. F. Boisvert, C. W. Clark, B. R. Miller, B. V. Saunders, H. S. Cohl, and M. A. McClain, http://dlmf .nist.gov/, Release 1.0.28 of 2020-09-15.

[55] N. Klco and M. J. Savage, Geometric quantum information structure in quantum fields and their lattice simulation, Phys. Rev. D 103, 065007 (2021).

[56] M. Horodecki, P. Horodecki, and R. Horodecki, MixedState Entanglement and Distillation: Is There a "Bound" Entanglement in Nature?, Phys. Rev. Lett. 80, 5239 (1998).

[57] C. H. Bennett, D. P. Divincenzo, T. Mor, P. W. Shor, J. A. Smolin, and B. M. Terhal, Unextendible Product Bases and Bound Entanglement, Phys. Rev. Lett. 82, 5385 (1999).

[58] D. Bruß and A. Peres, Construction of quantum states with bound entanglement, Phys. Rev. A 61, 030301(R) (2000).

[59] P. Horodecki and M. Lewenstein, Bound Entanglement and Continuous Variables, Phys. Rev. Lett. 85, 2657 (2000).

[60] R. F. Werner and M. M. Wolf, Bound Entangled Gaussian States, Phys. Rev. Lett. 86, 3658 (2001).

[61] J. A. Smolin, Four-party unlockable bound entangled state, Phys. Rev. A 63, 032306 (2001).

[62] H.-P. Breuer, Optimal Entanglement Criterion for Mixed Quantum States, Phys. Rev. Lett. 97, 080501 (2006).

[63] K. M. R. Audenaert, J. Eisert, and M. B. Plenio, Entanglement in systems of interacting harmonic oscillators, in Quantum Information with Continuous Variables of Atoms and Light, edited by N. J. Cerf, G. Leuchs, and E. S. Polzik (Imperial College Press, London, 2007), pp. 43-62.

[64] H. Wang, M. P. Blencowe, C. M. Wilson, and A. J. Rimberg, Mechanically generating entangled photons from the vacuum: A microwave circuit-acoustic resonator analog of the oscillatory Unruh effect, Phys. Rev. A 99, 053833 (2019).
[65] E. T. Holland, K. A. Wendt, K. Kravvaris, X. Wu, W. E. Ormand, J. L DuBois, S. Quaglioni, and F. Pederiva, Optimal Control for the quantum simulation of nuclear dynamics, Phys. Rev. A 101, 062307 (2020).

[66] A. Roy, D. Schuricht, J. Hauschild, F. Pollmann, and H. Saleur, The quantum sine-Gordon model with quantum circuits, Nucl. Phys. B968, 115445 (2021).

[67] J. S. C. Hung, J. H. Busnaina, C. W. Sandbo Chang, A. M. Vadiraj, I. Nsanzineza, E. Solano, H. Alaeian, E. Rico, and C. M. Wilson, Quantum Simulation of the Bosonic Creutz Ladder with a Parametric Cavity, Phys. Rev. Lett. 127, 100503 (2021).

[68] S. R. Beane, D. B. Kaplan, N. Klco, and M. J. Savage, Entanglement Suppression and Emergent Symmetries of Strong Interactions, Phys. Rev. Lett. 122, 102001 (2019).

[69] S. Fleming, T. Mehen, and I. W. Stewart, NNLO corrections to nucleon-nucleon scattering and perturbative pions, Nucl. Phys. A677, 313 (2000).

[70] S. R. Beane, P. F. Bedaque, M. J. Savage, and U. van Kolck, Towards a perturbative theory of nuclear forces, Nucl. Phys. A700, 377 (2002).

[71] P. Kunkel, M. Prüfer, H. Strobel, D. Linnemann, A. Frölian, T. Gasenzer, M. Gärttner, and M. K. Oberthaler, Spatially distributed multipartite entanglement enables EPR steering of atomic clouds, Science 360, 413 (2018).

[72] N. Sánchez-Kuntz and S. Floerchinger, Spatial entanglement in interacting Bose-Einstein condensates, Phys. Rev. A 103, 043327 (2021).

[73] D. E. Kharzeev and E. M. Levin, Deep inelastic scattering as a probe of entanglement, Phys. Rev. D 95, 114008 (2017).

[74] O. K. Baker and D. E. Kharzeev, Thermal radiation and entanglement in proton-proton collisions at energies available at the CERN Large Hadron Collider, Phys. Rev. D 98, 054007 (2018).

[75] J. Berges, S. Floerchinger, and R. Venugopalan, Entanglement and thermalization, Nucl. Phys. A982, 819 (2019).

[76] Z. Tu, D. E. Kharzeev, and T. Ullrich, Einstein-PodolskyRosen Paradox and Quantum Entanglement at Subnucleonic Scales, Phys. Rev. Lett. 124, 062001 (2020).

[77] D. E. Kharzeev and E. Levin, Deep inelastic scattering as a probe of entanglement: Confronting experimental data, Phys. Rev. D 104, L031503 (2021).

[78] Wolfram Research, Inc., Mathematica, Version 11.1, Champaign, IL, 2020.

[79] Advanpix LLC, Multiprecision computing toolbox for MATLAB, https://www.advanpix.com/.

[80] MATLAB, version 9.8.0.1396136 (R2020a) Update 3 (The MathWorks, Inc., Natick, Massachusetts, 2020). 